\title{
Causal Construction of the Massless Vertex Diagram
}

\author{
ANDREAS ASTE \\ Department of Physics and Astronomy, Theory Division, University of Basel, \\ Klingelbergstrasse 82, 4056 Basel, Switzerland. e-mail: andreas.aste@unibas.ch
}

Received: 17 July 2006; revised version: 25 August 2006

Published online: 28 September 2006

\begin{abstract}
The massless one-loop vertex diagram is constructed by exploiting the causal structure of the diagram in configuration space, which can be translated directly into dispersive relations in momentum space.
\end{abstract}

Mathematics Subject Classification (2000). 81T05, 81T15, $81 \mathrm{~T} 18$.

Keywords. causality, perturbative calculations, Feynman diagrams.

\section{Introduction}

In the traditional approach to quantum field theory, one starts from classical fields and a Lagrangean which describes the interaction. These objects get quantized and $S$-matrix elements or Greens functions are constructed with the help of the Feynman rules. A typical example which is used in this paper as a model theory is the massless $\Phi^{3}$-theory, where the interaction Hamiltonian density is given by the normal-ordered third-order monomial of a free uncharged massless scalar field and a coupling constant $\lambda$

$$
\mathcal{H}_{\text {int }}(x)=\frac{\lambda}{3 !}: \Phi(x)^{3}:
$$

and $x$ is an element of Minkowski space, denoted by $\mathbb{R}^{4}$ in the following. The perturbative $S$-Matrix is then constructed according to the expansion

$$
S=\mathbf{1}+\sum_{n=1}^{\infty} \frac{(-i)^{n}}{n !} \int \mathrm{d} x_{1}^{4} \cdots \mathrm{d} x_{n}^{4} T\left\{\mathcal{H}_{\text {int }}\left(x_{1}\right) \mathcal{H}_{\text {int }}\left(x_{2}\right) \cdots \mathcal{H}_{\text {int }}\left(x_{n}\right)\right\}
$$

where $T$ is the time-ordering operator. It must be pointed out that the perturbation series Equation (2) is formal and it is difficult to make any statement about the convergence of this series. Furthermore, two problems arise in the expansion given above. First, the time-ordered products

$$
T_{n}\left(x_{1}, x_{2}, \ldots, x_{n}\right)=(-i)^{n} T\left\{\mathcal{H}_{i n t}\left(x_{1}\right) \mathcal{H}_{i n t}\left(x_{2}\right) \cdots \mathcal{H}_{i n t}\left(x_{n}\right)\right\}
$$


are usually plagued by ultraviolet divergences. However, these divergences can be removed by regularization, such that the operator-valued distributions $T_{n}$ can be viewed as well-defined, already regularized expressions. Second, infrared divergences are also present in Equation (2). This is not astonishing, since the $T_{n}$ 's are operator-valued distributions, and therefore must be smeared out by test functions in $\mathcal{S}\left(\mathbb{R}^{4 n}\right)$, the Schwartz space of functions of rapid decrease. One may therefore introduce a test function $g(x) \in \mathcal{S}\left(\mathbb{R}^{4}\right)$ which plays the role of an 'adiabatic switching' and provides a cutoff in the long-range part of the interaction, which can be considered as a natural infrared regulator $[1,2]$. Then the infrared regularized $S$-matrix is given by

$$
S(g)=\mathbf{1}+\sum_{n=1}^{\infty} \frac{1}{n !} \int \mathrm{d} x_{1}^{4} \cdots \mathrm{d} x_{n}^{4} T_{n}\left(x_{1}, \ldots, x_{n}\right) g\left(x_{1}\right) \cdots g\left(x_{n}\right),
$$

and an appropriate adiabatic limit $g \rightarrow 1$ must be performed at the end of actual calculations in the right quantities (like cross-sections) where this limit exists. This is not one of the standard strategies usually found in the literature; however, it is the most natural one in view of the mathematical framework used in perturbative quantum field theory.

The vertex diagram appears in $\Phi^{3}$-theory at third-order of perturbation theory via

$$
\begin{aligned}
T_{3}\left(x_{1}, x_{2}, x_{3}\right) & =\left(\frac{-i \lambda}{3 !}\right)^{3} T\left\{: \Phi^{3}\left(x_{1}\right):: \Phi^{3}\left(x_{2}\right):: \Phi^{3}\left(x_{3}\right):\right\} \\
& =\lambda^{3} V\left(x_{1}, x_{2}, x_{3}\right): \Phi\left(x_{1}\right) \Phi\left(x_{2}\right) \Phi\left(x_{3}\right):+ \text { other graphs }
\end{aligned}
$$

where $V\left(x_{1}, x_{2}, x_{3}\right)=\Delta_{F}\left(x_{1}-x_{3}\right) \Delta_{F}\left(x_{3}-x_{2}\right) \Delta_{F}\left(x_{2}-x_{1}\right)$ is given by the scalar Feynman propagator discussed below in further detail. Of course, the massless vertex functions plays also a prominent role in applied quantum field theories like QCD, where it emerges in the three-gluon vertex [3], or in perturbative models of quantum gravity.

In this paper, the Fourier transform of the massless vertex distribution (also called vertex function in the sequel)

$$
\begin{aligned}
\hat{V}(p, q) & =\int \mathrm{d}^{4} x_{1} \mathrm{~d}^{4} x_{2} V\left(x_{1}, x_{2}, x_{3}\right) \mathrm{e}^{i p\left(x_{1}-x_{3}\right)+i q\left(x_{3}-x_{2}\right)} \\
& =(2 \pi)^{-4} \int \frac{\mathrm{d}^{4} k}{\left[(p-k)^{2}+i 0\right]\left[(q-k)^{2}+i 0\right]\left[k^{2}+i 0\right]}
\end{aligned}
$$

will be calculated in a non-standard manner by making explicit use of causality as a fundamental property of local quantum field theories [4]. The presented dispersive method is based on considerations in configuration space and integrals in momentum space are not evaluated by the help of Wick rotations. Furthermore, no mass terms are needed as regulators of infrared divergences, the vertex function is constructed directly without any limiting procedure. 
It should be noted that the vertex function is translation invariant in the sense that $V\left(x_{1}, x_{2}, x_{3}\right)=V\left(x_{1}-a, x_{2}-a, x_{3}-a\right)$, where $a$ is an arbitrary four-vector. This symmetry property can be inferred directly from the decomposition of the vertex function into three Feynman propagators given above. Introducing new integration variables $z_{1}=x_{1}-x_{3}$ and $z_{2}=x_{3}-x_{2}$, the Fourier transform in Equation (6) can also be written as $\left(\Delta_{F}(x)=\Delta_{F}(-x)\right)$

$$
\begin{aligned}
\hat{V}(p, q) & =\int \mathrm{d}^{4} z_{1} \mathrm{~d}^{4} z_{2} V\left(z_{1}+x_{3},-z_{2}+x_{3}, x_{3}\right) \mathrm{e}^{i p z_{1}+i q z_{2}} \\
& =\int \mathrm{d}^{4} z_{1} \mathrm{~d}^{4} z_{2} \Delta_{F}\left(z_{1}\right) \Delta_{F}\left(z_{2}\right) \Delta_{F}\left(z_{1}+z_{2}\right) \mathrm{e}^{i p z_{1}+i q z_{2}},
\end{aligned}
$$

which shows that $\hat{V}(p, q)$ does indeed not depend on $x_{3}$.

The focus of this work is on the causal and analytic properties of the vertex function and its infrared-finite dispersive calculation in the massless case. The more involved general-mass case of the vertex function has been treated by 't Hooft and Veltman [5], such that results for the massless case could be obtained by considering the appropriate limit. An explicit result for the massless case has been given in [6], and a discussion of the vertex function within a special framework based on a geometrical interpretation of kinematic invariants and other quantities of one-loop diagrams is contained in [7], where a short overview over different calculational methods for one-loop $N$-point functions can also be found.

Before investigating the causal structure of the scalar vertex function, some important analytic properties of the Feynman propagator and other related tempered distributions will be recalled in the following section.

\section{Properties of Tempered Distributions}

The Feynman propagator of a free scalar and charge neutral quantum field $\Phi(x)$ fulfilling the wave equation

$$
\square \Phi(x)=\partial_{\mu} \partial^{\mu} \Phi(x)=0
$$

is given in configuration space by the vacuum expectation value

$$
\begin{aligned}
\Delta_{F}(x) & =\Delta_{F}\left(x_{1}-x_{2}\right)=-i\langle 0|T(\Phi(x) \Phi(0))| 0\rangle=-i\left\langle 0\left|T\left(\Phi\left(x_{1}\right) \Phi\left(x_{2}\right)\right)\right| 0\right\rangle \\
& =\int \frac{\mathrm{d}^{4} k}{(2 \pi)^{4}} \frac{\mathrm{e}^{-i k x}}{k^{2}+i 0}=\frac{i}{4 \pi^{2}} \frac{1}{x^{2}-i 0}=\frac{i}{4 \pi^{2}} P \frac{1}{x^{2}}-\frac{1}{4 \pi} \delta\left(x^{2}\right),
\end{aligned}
$$

where $P$ denotes principal value regularization and $\delta$ is the one-dimensional Dirac distribution depending on $x^{2}=x_{\mu} x^{\mu}=\left(x^{0}\right)^{2}-\left(x^{1}\right)^{2}-\left(x^{2}\right)^{2}-\left(x^{3}\right)^{2}=x_{0}^{2}-\vec{x}^{2}$ [8].

Knowledge of the basic properties of the Feynman propagator is sufficient for many applications in perturbative quantum field theory. For the forthcoming discussion, it is instructive to review some of the useful properties of basic tempered 
distributions appearing in quantum field theory. A scalar neutral field $\Phi$ can be decomposed into a negative and positive frequency part according to

$$
\Phi(x)=\Phi^{-}(x)+\Phi^{+}(x)=\int \frac{\mathrm{d}^{3} k}{\sqrt{(2 \pi)^{3} 2|\vec{k}|}}\left[a(\vec{k}) \mathrm{e}^{-i k x}+a^{\dagger}(\vec{k}) \mathrm{e}^{i k x}\right]
$$

with

$$
\left[a(\vec{k}), a^{\dagger}\left(\vec{k}^{\prime}\right)\right]=\delta^{(3)}\left(\vec{k}-\vec{k}^{\prime}\right), \quad\left[a(\vec{k}), a\left(\vec{k}^{\prime}\right)\right]=\left[a^{\dagger}(\vec{k}), a^{\dagger}\left(\vec{k}^{\prime}\right)\right]=0 .
$$

The commutation relations for such a field are given by the positive and negative frequency Jordan-Pauli distributions

$$
\Delta^{ \pm}(x)=-i\left[\Phi^{\mp}(x), \quad \Phi^{ \pm}(0)\right]=-i\left\langle 0\left|\left[\Phi^{\mp}(x), \quad \Phi^{ \pm}(0)\right]\right| 0\right\rangle,
$$

which have the Fourier transforms

$$
\hat{\Delta}^{ \pm}(k)=\int \mathrm{d}^{4} x \Delta^{ \pm}(x) \mathrm{e}^{i k x}=\mp(2 \pi i) \Theta\left( \pm k^{0}\right) \delta\left(k^{2}\right) .
$$

The fact that the commutator

$$
[\Phi(x), \Phi(0)]=i \Delta^{+}(x)+i \Delta^{-}(x)=: i \Delta(x)
$$

with the Fourier transform

$$
\hat{\Delta}(k)=-(2 \pi i) \operatorname{sgn}\left(k^{0}\right) \delta\left(k^{2}\right)
$$

vanishes for spacelike arguments $x^{2}<0$ due to the requirement of microcausality, leads to the important property that the Jordan-Pauli distribution $\Delta$ has causal support, i.e. it vanishes outside the closed forward and backward light cone such that

$$
\operatorname{supp} \Delta(x) \subseteq \bar{V}^{-} \cup \bar{V}^{+}, \quad \bar{V}^{ \pm}=\left\{x \mid x^{2} \geq 0, \pm x^{0} \geq 0\right\}
$$

in the sense of distributions. A further crucial observation is the fact that one can introduce the retarded propagator $\Delta^{\text {ret }}(x)$ which coincides with $\Delta(x)$ on $\bar{V}^{+}-\{0\}$, i.e. $\left\langle\Delta^{\text {ret }}, \varphi\right\rangle=\langle\Delta, \varphi\rangle$ holds for all test functions in the Schwartz space $\varphi \in \mathcal{S}\left(\mathbb{R}^{4}\right)$ with support $\operatorname{supp} \varphi \subset \mathbb{R}^{4}-\bar{V}^{-}$. One may write in configuration space

$$
\Delta^{\mathrm{ret}}(x)=\Theta\left(x^{0}\right) \Delta(x),
$$

and transform this 'splitting' formula into a convolution in momentum space ${ }^{1}$

$$
\hat{\Delta}^{\mathrm{ret}}(k)=\int \frac{\mathrm{d}^{4} p}{(2 \pi)^{4}} \hat{\Delta}(p) \hat{\Theta}(k-p) .
$$

\footnotetext{
${ }^{1}$ The Heaviside distribution $\Theta\left(x^{0}\right)$ could be replaced by $\Theta(v x)$ with an arbitrary vector $v \in$ $V^{+}=\bar{V}^{+}-\partial \bar{V}^{+}$inside the open forward light cone.
} 
The Fourier transform of the Heaviside distribution $\Theta\left(x^{0}\right)$ can be calculated easily

$$
\hat{\Theta}(k)=\lim _{\epsilon \rightarrow 0} \int \mathrm{d}^{4} x \Theta\left(x^{0}\right) \mathrm{e}^{-\epsilon x^{0}} \mathrm{e}^{i k_{0} x^{0}-i \vec{k} \vec{x}}=\frac{(2 \pi)^{3} i}{k^{0}+i 0} \delta^{(3)}(\vec{k}) .
$$

For the special case where $k$ is in the forward light cone $V^{+}$, one can go to a Lorentz frame where $k=\left(k^{0}, \overrightarrow{0}\right)$ such that Equation (18) becomes

$$
\hat{\Delta}^{\mathrm{ret}}\left(k^{0}, \overrightarrow{0}\right)=\frac{i}{2 \pi} \int \mathrm{d} p^{0} \frac{\hat{\Delta}\left(\left(p^{0}, \overrightarrow{0}\right)\right)}{k^{0}-p^{0}+i 0}=\frac{i}{2 \pi} \int \mathrm{d} t \frac{\hat{\Delta}\left(\left(t k^{0}, \overrightarrow{0}\right)\right)}{1-t+i 0} .
$$

Hence, for arbitrary $k \in V^{+}, \hat{\Delta}^{\text {ret }}$ would be given by the dispersion relation

$$
\hat{\Delta}^{\mathrm{ret}}(k)=\frac{i}{2 \pi} \int \mathrm{d} t \frac{\hat{\Delta}(t k)}{1-t+i 0} .
$$

However, the integral in Equation (21) is undefined in the massless case considered here. This illustrates the fact that it is not possible in general to convert the product of two distributions into a convolution via a Fourier transform, even though expression (17) describes a well-defined distribution. Here, one can circumvent this problem, e.g., by introducing a mass for the field $\Phi$ as a regulator for the corresponding massive Jordan-Pauli distribution which is also defined as a field commutator (for a different method, see, e.g., [9]). For the massive Jordan-Pauli distribution $\Delta_{m}(x)$ one gets in momentum space

$$
\hat{\Delta}_{m}(k)=-(2 \pi i) \operatorname{sgn}\left(k^{0}\right) \delta\left(k^{2}-m^{2}\right)
$$

and the following expression for the massive retarded distribution $\hat{\Delta}_{m}^{\text {ret }}(k)\left(k \in V^{+}\right)$

$$
\begin{aligned}
\hat{\Delta}_{m}^{\text {ret }}(k) & =\int \mathrm{d} t \frac{\operatorname{sgn}\left(t k^{0}\right) \delta\left(t^{2} k^{2}-m^{2}\right)}{1-t+i 0} \\
& =\int \mathrm{d} t \frac{\left[\delta\left(t-\frac{m}{\sqrt{k^{2}}}\right)-\delta\left(t+\frac{m}{\sqrt{k^{2}}}\right)\right]}{2 \sqrt{k^{2}} m(1-t+i 0)}=\frac{1}{k^{2}-m^{2}} \quad\left(k^{2} \neq m^{2}\right) .
\end{aligned}
$$

The $\delta$-distribution in the Fourier transformed Jordan-Pauli distribution Equation (22) contains a mass term which is not present in Equation (15), such that $\hat{\Delta}_{m}(k)$ does not exhibit a singular behavior on the light cone in momentum space.

As a special case of the edge of the wedge theorem [4] it is known that the Fourier transform of the retarded distribution $\Delta^{\text {ret }}(x)$ is the boundary value of an analytic function $r(z)$, regular in $T^{+}:=\mathbb{R}^{4}+i V^{+}[1,10]$. This way one obtains from $r(z)=1 /\left(z^{2}-m^{2}\right), z \in T^{+}$, and $m \rightarrow 0$

$$
\hat{\Delta}^{\mathrm{ret}}(k)=\frac{1}{k^{2}+i k^{0} 0}=\frac{1}{k^{2}+i 0}+2 \pi i \Theta\left(-k^{0}\right) \delta\left(k^{2}\right) .
$$

The analytic expression for the Feynman propagator is then recovered, and one obtains

$$
\hat{\Delta}^{\mathrm{ret}}(k)=\hat{\Delta}_{F}(k)+\hat{\Delta}^{-}(k) .
$$


It is helpful for the forthcoming to understand Equation (25). This is best done in configuration space. One obviously has

$$
\begin{aligned}
i \Delta_{F}\left(x_{1}-x_{2}\right) & =\left\langle 0\left|T\left(\Phi\left(x_{1}\right) \Phi\left(x_{2}\right)\right)\right| 0\right\rangle \\
& =\left\langle 0\left|\Theta\left(x_{1}^{0}-x_{2}^{0}\right) \Phi\left(x_{1}\right) \Phi\left(x_{2}\right)\right| 0\right\rangle+\left\langle 0\left|\Theta\left(x_{2}^{0}-x_{1}^{0}\right) \Phi\left(x_{2}\right) \Phi\left(x_{1}\right)\right| 0\right\rangle
\end{aligned}
$$

and

$$
\begin{aligned}
i \Delta^{-}\left(x_{1}-x_{2}\right) & =-\left\langle 0\left|\Phi\left(x_{2}\right) \Phi\left(x_{1}\right)\right| 0\right\rangle \\
& =-\left\langle 0\left|\Theta\left(x_{2}^{0}-x_{1}^{0}\right) \Phi\left(x_{2}\right) \Phi\left(x_{1}\right)\right| 0\right\rangle-\left\langle 0\left|\Theta\left(x_{1}^{0}-x_{2}^{0}\right) \Phi\left(x_{2}\right) \Phi\left(x_{1}\right)\right| 0\right\rangle,
\end{aligned}
$$

and therefore Equation (24) is fulfilled

$$
\begin{aligned}
i \Delta^{\mathrm{ret}}\left(x_{1}-x_{2}\right) & =i \Theta\left(x_{1}^{0}-x_{2}^{0}\right) \Delta\left(x_{1}-x_{2}\right) \\
& =\left\langle 0\left|\Theta\left(x_{1}^{0}-x_{2}^{0}\right) \Phi\left(x_{1}\right) \Phi\left(x_{2}\right)\right| 0\right\rangle-\left\langle 0\left|\Theta\left(x_{1}^{0}-x_{2}^{0}\right) \Phi\left(x_{2}\right) \Phi\left(x_{1}\right)\right| 0\right\rangle .
\end{aligned}
$$

The discussion presented here can be applied the same way to second order loop diagrams [11-13]. The problem of ultraviolet divergences can be solved in such cases by using subtracted dispersion relations [1].

\section{Causality}

The causal properties of the Jordan-Pauli distribution and the retarded propagator can be generalized in an analogous manner to the case of the vertex function. For this purpose, one defines the causal operator-valued three-point functions $\Delta_{3, \mathrm{op}}^{ \pm}, \Delta_{3}^{\mathrm{op}}$ and related C-number valued contractions $\Delta_{3}^{ \pm}$and $\Delta_{3}$, which are related to the vertex diagram via

$$
\begin{aligned}
\Delta_{3, \mathrm{op}}^{-}\left(x_{1}, x_{2}, x_{3}\right)= & -T_{2}\left(x_{2}, x_{3}\right) T_{1}\left(x_{1}\right)-T_{1}\left(x_{3}\right) T_{2}\left(x_{1}, x_{2}\right)-T_{2}\left(x_{1}, x_{3}\right) T_{1}\left(x_{2}\right)+ \\
& +T_{1}\left(x_{3}\right) T_{1}\left(x_{1}\right) T_{1}\left(x_{2}\right)+T_{1}\left(x_{3}\right) T_{1}\left(x_{2}\right) T_{1}\left(x_{1}\right) \\
= & \lambda^{3} \Delta_{3}^{-}\left(x_{1}, x_{2}, x_{3}\right): \Phi\left(x_{1}\right) \Phi\left(x_{2}\right) \Phi\left(x_{3}\right): \text { other graphs, } \\
\Delta_{3, o p}^{+}\left(x_{1}, x_{2}, x_{3}\right)= & +T_{1}\left(x_{1}\right) T_{2}\left(x_{2}, x_{3}\right)+T_{2}\left(x_{1}, x_{2}\right) T_{1}\left(x_{3}\right)+T_{1}\left(x_{2}\right) T_{2}\left(x_{1}, x_{3}\right) \\
& -T_{1}\left(x_{1}\right) T_{1}\left(x_{2}\right) T_{1}\left(x_{3}\right)-T_{1}\left(x_{2}\right) T_{1}\left(x_{1}\right) T_{1}\left(x_{3}\right) \\
= & \lambda^{3} \Delta_{3}^{+}\left(x_{1}, x_{2}, x_{3}\right): \Phi\left(x_{1}\right) \Phi\left(x_{2}\right) \Phi\left(x_{3}\right): \text { +other graphs, }
\end{aligned}
$$

and

$$
\begin{aligned}
\Delta_{3}^{\mathrm{op}} & \left(x_{1}, x_{2}, x_{3}\right)=\Delta_{3, \mathrm{op}}^{+}\left(x_{1}, x_{2}, x_{3}\right)+\Delta_{3, \mathrm{op}}^{-}\left(x_{1}, x_{2}, x_{3}\right) \\
= & {\left[T_{1}\left(x_{1}\right), T_{2}\left(x_{2}, x_{3}\right)\right]+\left[T_{1}\left(x_{2}\right), T_{2}\left(x_{1}, x_{3}\right)\right]-\left[T_{1}\left(x_{3}\right), T_{2}\left(x_{1}, x_{2}\right)\right] } \\
& +\left[T_{1}\left(x_{3}\right), T_{1}\left(x_{1}\right) T_{1}\left(x_{2}\right)\right]+\left[T_{1}\left(x_{3}\right), T_{1}\left(x_{2}\right) T_{1}\left(x_{1}\right)\right] \\
= & \lambda^{3} \Delta_{3}\left(x_{1}, x_{2}, x_{3}\right): \Phi\left(x_{1}\right) \Phi\left(x_{2}\right) \Phi\left(x_{3}\right):+ \text { other graphs, }
\end{aligned}
$$

where $\Delta_{3}^{\mathrm{op}}$ and $\Delta_{3}$ have causal support as distributions in the sense that 


$$
\operatorname{supp} \Delta_{3}^{(\mathrm{op})}\left(x_{1}, x_{2}, x_{3}\right) \subseteq \Gamma_{3}^{+} \cup \Gamma_{3}^{-},
$$

where

$$
\Gamma_{n}^{ \pm}=\left\{\left(x_{1}, \ldots, x_{n}\right) \mid x_{n} \in \mathbb{R}^{4}, \quad\left(x_{j}-x_{n}\right) \in \bar{V}^{ \pm} \forall j=1, \ldots, n-1\right\} .
$$

Therefore, only when both arguments $x_{1}$ and $x_{2}$ are in the forward light cone or both arguments $x_{1}$ and $x_{2}$ are in the backward light cone with respect to $x_{3}$, $\left(x_{1}, x_{2}, x_{3}\right)$ belongs to the support of $\Delta_{3}^{(\mathrm{op})}$. For the sake of brevity, only a short outline how one can prove the special support property of $\Delta_{3}^{\text {op }}$ is given here by considering the case where, e.g., $x_{1} \in \bar{V}^{+}\left(x_{3}\right)$ and $x_{2} \notin \bar{V}^{+}\left(x_{3}\right)$. In this case, one can assume that one is in a Lorentz system where $x_{1}^{0}>x_{3}^{0}>x_{2}^{0}$, since the transformation properties of all $T_{n}$ 's are fixed by the representation of the Lorentz group on the Fock space of massless scalar particles. Then one has, e.g., $T_{2}\left(x_{2}, x_{3}\right)=T\left(T_{1}\left(x_{2}\right) T_{1}\left(x_{3}\right)\right)=T_{1}\left(x_{3}\right) T_{1}\left(x_{2}\right)$, and accordingly for the full expression Equation (31)

$$
\begin{aligned}
\Delta_{3}^{\mathrm{op}}\left(x_{1}, x_{2}, x_{3}\right)= & +T_{1}\left(x_{1}\right) T_{1}\left(x_{3}\right) T_{1}\left(x_{2}\right)-T_{1}\left(x_{3}\right) T_{1}\left(x_{2}\right) T_{1}\left(x_{1}\right)+ \\
& +T_{1}\left(x_{2}\right) T_{1}\left(x_{1}\right) T_{1}\left(x_{3}\right)-T_{1}\left(x_{1}\right) T_{1}\left(x_{3}\right) T_{1}\left(x_{2}\right)+ \\
& -T_{1}\left(x_{3}\right) T_{1}\left(x_{1}\right) T_{1}\left(x_{2}\right)+T_{1}\left(x_{1}\right) T_{1}\left(x_{2}\right) T_{1}\left(x_{3}\right)+ \\
& +T_{1}\left(x_{3}\right) T_{1}\left(x_{1}\right) T_{1}\left(x_{2}\right)-T_{1}\left(x_{1}\right) T_{1}\left(x_{2}\right) T_{1}\left(x_{3}\right)+ \\
& +T_{1}\left(x_{3}\right) T_{1}\left(x_{2}\right) T_{1}\left(x_{1}\right)-T_{1}\left(x_{2}\right) T_{1}\left(x_{1}\right) T_{1}\left(x_{3}\right)=0 .
\end{aligned}
$$

The same way one may check for all other cases that $\Delta_{3}^{\mathrm{op}}$ vanishes whenever the causal condition (32) is not fulfilled. The general definition of causal distributions $\Delta_{n}^{\mathrm{op}}\left(x_{1}, \ldots, x_{n}\right)$ at higher orders can be found in $[1,11]$.

One should point out that $x_{3}$ plays a distinguished role in the discussion above. Whereas the time-ordered distribution $T_{3}\left(x_{1}, x_{2}, x_{3}\right)=T_{3}\left(x_{i_{1}}, x_{i_{2}}, x_{i_{3}}\right)$ is invariant under a permutation $(1,2,3) \rightarrow\left(i_{1}, i_{2}, i_{3}\right)$ of the arguments according to its definition in Equations $(2,3,4), \Delta_{3}^{\mathrm{op}}\left(x_{1}, x_{2}, x_{3}\right)$ is not. However, the construction presented above would go through with $x_{1}$ or $x_{2}$ as distinguished arguments. The crucial point is that the causal support property of $\Delta_{3}^{\mathrm{op}}$ and its C-number part $\Delta_{3}$ will enable the calculation of a distribution which is retarded with respect to $x_{3}$ via a double dispersion relation in momentum space, as will be shown in the following. This retarded distribution $\hat{\Delta}_{3}^{\text {ret }}$ can be related directly to the vertex function in analogy to Equation (25). The vertex function again possesses the full permutation symmetry in configuration space by construction.

The vertex function will be constructed below by performing the following calculational steps. The Fourier transform $\hat{\Delta}_{3}(p, q)=\int \mathrm{d}^{4} x_{1} \mathrm{~d}^{4} x_{2} \Delta_{3}\left(x_{1}, x_{2}, x_{3}\right)$ $\mathrm{e}^{i p\left(x_{1}-x_{3}\right)+i q\left(x_{3}-x_{2}\right)}$, which is independent of $x_{3}$ due to translation invariance (see also Chapter 3.1 in [1]), is calculated from the expression Equation (31) in configuration space. Then the corresponding retarded distribution $\Delta_{3}^{\text {ret }}\left(x_{1}, x_{2}, x_{3}\right)$ $=\Theta\left(x_{1}-x_{3}\right) \Theta\left(x_{2}-x_{3}\right) \Delta_{3}\left(x_{1}, x_{2}, x_{3}\right)$ is evaluated in momentum space by a dispersion relation analogously to Equation (21), and finally the vertex function is 
obtained directly from analytic considerations. It is also straightforward to check that on the operator level, the time-ordered product $T_{3}$ can be obtained from the retarded (appropriately regularized) distribution $\Delta_{3}^{\mathrm{op}, \text { ret }}\left(x_{1}, x_{2}, x_{3}\right)$

$$
T_{3}\left(x_{1}, x_{2}, x_{3}\right)=\Delta_{3}^{\mathrm{op}, \text { ret }}\left(x_{1}, x_{2}, x_{3}\right)-\Delta_{3, \mathrm{op}}^{-}\left(x_{1}, x_{2}, x_{3}\right),
$$

i.e. for the vertex distribution holds

$$
V\left(x_{1}, x_{2}, x_{3}\right)=\Delta_{3}^{\text {ret }}\left(x_{1}, x_{2}, x_{3}\right)-\Delta_{3}^{-}\left(x_{1}, x_{2}, x_{3}\right)
$$

in close analogy to Equation (25).

\section{Calculation of $\hat{\boldsymbol{\Delta}}_{3}$}

Starting from the first- and second-order tree diagram given by

$$
\begin{aligned}
T_{2}\left(x_{1}, x_{2}\right) & =(-i)^{2} \frac{\lambda^{2}}{(3 !)^{2}} T: \Phi^{3}\left(x_{1}\right):: \Phi^{3}\left(x_{2}\right) \\
& :=-i \frac{\lambda^{2}}{4}: \Phi^{2}\left(x_{1}\right) \Phi^{2}\left(x_{2}\right): \Delta_{F}\left(x_{1}-x_{2}\right)+\text { other graphs, }
\end{aligned}
$$

one readily obtains for $\Delta_{3}$ in configuration space by exploiting the relevant Wick contractions in Equation (31)

$$
\begin{aligned}
& \Delta_{3}\left(x_{1}, x_{2}, x_{3}\right)=\Delta_{3}^{+}\left(x_{1}, x_{2}, x_{3}\right)+\Delta_{3}^{-}\left(x_{1}, x_{2}, x_{3}\right) \\
&=+\Delta^{+}\left(x_{1}-x_{2}\right) \Delta^{+}\left(x_{1}-x_{3}\right) \Delta_{F}\left(x_{2}-x_{3}\right)-\Delta^{+}\left(x_{2}-x_{1}\right) \Delta^{+}\left(x_{3}-x_{1}\right) \Delta_{F}\left(x_{2}-x_{3}\right)+ \\
&+\Delta^{+}\left(x_{2}-x_{1}\right) \Delta^{+}\left(x_{2}-x_{3}\right) \Delta_{F}\left(x_{1}-x_{3}\right)-\Delta^{+}\left(x_{1}-x_{2}\right) \Delta^{+}\left(x_{3}-x_{2}\right) \Delta_{F}\left(x_{1}-x_{3}\right)+ \\
&+\Delta_{F}\left(x_{1}-x_{2}\right) \Delta^{+}\left(x_{1}-x_{3}\right) \Delta^{+}\left(x_{2}-x_{3}\right)-\Delta_{F}\left(x_{1}-x_{2}\right) \Delta^{+}\left(x_{3}-x_{1}\right) \Delta^{+}\left(x_{3}-x_{2}\right)+ \\
&+\Delta^{+}\left(x_{3}-x_{1}\right) \Delta^{+}\left(x_{3}-x_{2}\right) \Delta^{+}\left(x_{1}-x_{2}\right)-\Delta^{+}\left(x_{1}-x_{3}\right) \Delta^{+}\left(x_{2}-x_{3}\right) \Delta^{+}\left(x_{1}-x_{2}\right)+ \\
&+\Delta^{+}\left(x_{3}-x_{1}\right) \Delta^{+}\left(x_{3}-x_{2}\right) \Delta^{+}\left(x_{2}-x_{1}\right)-\Delta^{+}\left(x_{1}-x_{3}\right) \Delta^{+}\left(x_{2}-x_{3}\right) \Delta^{+}\left(x_{2}-x_{1}\right)
\end{aligned}
$$

Note that a Wick contraction without time-ordering leads to a $\Delta^{+}$-distribution instead of a Feynman propagator. Making use of $\Delta^{+}(-x)=-\Delta^{-}(x)$ and introducing the advanced distribution $\Delta^{\text {av }}$ such that $\Delta(x)=\Delta^{\text {ret }}(x)-\Delta^{\text {av }}(x)$ and $\Delta^{\text {ret }}(-x)=-\Delta^{\text {av }}(x)$, Equation (38) can be simplified to

$$
\begin{aligned}
& \Delta_{3}\left(x_{1}, x_{2}, x_{3}\right) \\
&=+\Delta^{-}\left(x_{1}-x_{3}\right) \Delta^{+}\left(x_{3}-x_{2}\right) \Delta_{F}\left(x_{1}-x_{2}\right)+\Delta^{+}\left(x_{1}-x_{3}\right) \Delta^{\mathrm{ret}}\left(x_{3}-x_{2}\right) \Delta^{+}\left(x_{1}-x_{2}\right)+ \\
&+\Delta^{-}\left(x_{1}-x_{3}\right) \Delta^{\mathrm{av}}\left(x_{3}-x_{2}\right) \Delta^{+}\left(x_{2}-x_{1}\right)-\left\{\left(x_{1}, x_{2}, x_{3}\right) \leftrightarrow\left(-x_{2},-x_{1},-x_{3}\right)\right\} . \quad \text { (39) }
\end{aligned}
$$


Performing the Fourier transform, one obtains

$$
\begin{aligned}
\hat{\Delta}_{3}(p, q)= & \int \mathrm{d}^{4} x_{1} \mathrm{~d}^{4} x_{2} \Delta\left(x_{1}, x_{2}, x_{3}\right) \mathrm{e}^{i p\left(x_{1}-x_{3}\right)+i q\left(x_{3}-x_{2}\right)}=(2 \pi)^{-4} \int \mathrm{d}^{4} k \times \\
\times & {\left[\hat{\Delta}^{-}(p-k) \hat{\Delta}^{+}(q-k) \hat{\Delta}_{F}(k)+\hat{\Delta}^{+}(p-k) \hat{\Delta}^{\mathrm{ret}}(q-k) \hat{\Delta}^{+}(k)+\right.} \\
& \left.+\hat{\Delta}^{-}(p-k) \hat{\Delta}^{\mathrm{av}}(q-k) \hat{\Delta}^{+}(-k)\right]-\{p \leftrightarrow q\} .
\end{aligned}
$$

From the integral Equation (40) one immediately derives the following symmetry properties of $\hat{\Delta}_{3}$

$$
\begin{aligned}
& \hat{\Delta}_{3}(p, q)=-\hat{\Delta}_{3}(q, p)=\hat{\Delta}_{3}(-p,-q), \\
& \hat{\Delta}_{3}(-p, q)=\hat{\Delta}_{3}(-q, p) .
\end{aligned}
$$

As an illustration, the full calculation of the first Fourier integral appearing in Equation (40)

$$
\begin{aligned}
(2 \pi)^{-2} I_{1}(p, q)= & (2 \pi)^{-4} \int \mathrm{d}^{4} k\left[\hat{\Delta}^{-}(p-k) \hat{\Delta}^{+}(q-k) \hat{\Delta}_{F}(k)\right] \\
= & (2 \pi)^{-2} \int \mathrm{d}^{4} k \Theta\left(k^{0}-p^{0}\right) \delta\left((p-k)^{2}\right) \Theta\left(q^{0}-k^{0}\right) \times \\
& \times \delta\left((q-k)^{2}\right) \frac{1}{k^{2}+i 0}
\end{aligned}
$$

is presented here. Introducing $P=p-q$ and using a new integration variable $k^{\prime}=k-p$, one obtains

$$
I_{1}(p, q)=\int \frac{\mathrm{d}^{4} k^{\prime}}{\left(k^{\prime}+p\right)^{2}+i 0} \Theta\left(k^{\prime 0}\right) \delta\left(k^{\prime 2}\right) \Theta\left(-P^{0}-k^{\prime 0}\right) \delta\left(\left(P+k^{\prime}\right)^{2}\right) .
$$

The first $\Theta$ and $\delta$ distributions can be written as $\delta\left(k^{0}-|\vec{k}|\right) / 2|\vec{k}|\left(k^{\prime}\right.$ is replaced by $k$ in the following). Taking into account the second $\Theta$ distribution one easily derives $P^{0} \leq-k^{0} \leq 0$, otherwise integral above would vanish. For spacelike $P$, $P^{2}<0$, there exists a Lorentz frame with $P^{0}>0$ such that $I_{1}$ also vanishes. For timelike $P$, there exists a Lorentz frame where $\vec{P}=\overrightarrow{0}=\vec{p}-\vec{q}$. The last $\delta$ distribution in Equation (43) then implies $\left(P^{0}+k^{0}\right)^{2}-\vec{k}^{2}=0$, and combined with $k^{0}=|\vec{k}|$ from the $\delta$ distribution $\delta\left(k^{0}-|\vec{k}|\right)$ one can replace $k^{0}$ by $-P^{0} / 2$ in the Feynman propagator appearing in Equation (43) after performing the trivial integral over $k^{0}$. For timelike $P$, there remains to calculate with $\delta\left(\left(P^{0}+k^{0}\right)^{2}-\vec{k}^{2}\right)$ replaced by $\delta\left(P_{0}^{2}+2 P_{0}|\vec{k}|\right)=\delta\left(|\vec{k}|-\frac{\left|P^{0}\right|}{2}\right) / 2\left|P^{0}\right|$

$$
I_{1}(p, q)=\Theta\left(-P^{0}\right) \int \frac{\mathrm{d}^{3} k}{2|\vec{k}|} \frac{\delta\left(|\vec{k}|-\frac{\left|P^{0}\right|}{2}\right)}{2\left|P^{0}\right|\left[(k+p)^{2}+i 0\right]},
$$

with $k$ in the denominator above given by $k=\left(-P^{0} / 2, \vec{k}\right)$, such that one can write

$$
(k+p)^{2}=\left(k^{0}+p^{0}\right)^{2}-(\vec{k}+\vec{p})^{2}=\left(-\frac{1}{2} P^{0}+p^{0}\right)^{2}-\vec{k}^{2}-\vec{p}^{2}-2|\vec{k}||\vec{p}| \cos \vartheta,
$$


and one has $|\vec{k}|=\left|P^{0}\right| / 2$. Hence $\left(P^{0}=P_{0}\right)$,

$$
\begin{aligned}
I_{1}(p, q) & =\frac{2 \pi}{8} \Theta\left(-P^{0}\right) \Theta\left(P_{0}^{2}\right) \int_{-1}^{+1} \frac{\mathrm{d} \cos \vartheta}{\left(-P^{0} / 2+p^{0}\right)^{2}-\vec{k}^{2}-\vec{p}^{2}-2|\vec{k}||\vec{p}| \cos \vartheta+i 0} \\
& =-\frac{\pi}{4} \frac{\Theta\left(-P^{0}\right) \Theta\left(P_{0}^{2}\right)}{\left|P^{0}\right||\vec{p}|} \log \left(\frac{\left(-P^{0} / 2+p^{0}\right)^{2}-(|\vec{k}|+|\vec{p}|)^{2}+i 0}{\left(-P^{0} / 2+p^{0}\right)^{2}-(|\vec{k}|-|\vec{p}|)^{2}+i 0}\right) \\
& =-\frac{\pi}{4} \frac{\Theta\left(-P^{0}\right) \Theta\left(P_{0}^{2}\right)}{\left|P^{0}\right||\vec{p}|} \log \left(\frac{p^{2}-P^{0} p^{0}-|\vec{p}|\left|P^{0}\right|+i 0}{p^{2}-P^{0} p^{0}+|\vec{p}|\left|P^{0}\right|+i 0}\right) .
\end{aligned}
$$

The distribution $\Theta\left(P_{0}^{2}\right)$ inserted in Equation (46) does not affect the result in the rest frame of $P$, but will account below for the fact that $I_{1}(p, q)$ vanishes for spacelike $P$ when written in covariant form $\Theta\left(P^{2}\right)$. Investigating the behavior of the expression $\left|\vec{p} \| P^{0}\right|$ under Lorentz transformations one finds that the covariant form of $\left|\vec{p} \| P^{0}\right|$ is given by $\sqrt{N}$, where $N$ is the triangle function

$$
N \equiv(P p)^{2}-P^{2} p^{2}=(p q)^{2}-p^{2} q^{2},
$$

such that one arrives at

$$
\begin{aligned}
I_{1}(p, q) & =-\frac{\pi}{4} \frac{\Theta\left(-P^{0}\right) \Theta\left(P^{2}\right)}{\sqrt{N}} \log \left(\frac{p q-\sqrt{N}+i 0}{p q+\sqrt{N}+i 0}\right) \\
& =\frac{\pi}{4} \frac{\Theta\left(-P^{0}\right) \Theta\left(P^{2}\right)}{\sqrt{N}} \log \left(\frac{p q+\sqrt{N}}{p q-\sqrt{N}}-i 0\right) .
\end{aligned}
$$

It is left to the reader as a simple exercise to show that lightlike $P$ do not contribute to $I_{1}(p, q)$ in a distributional sense. Calculating the remaining two integrals along the same lines as above and subtracting the resulting expressions with $p$ and $q$ interchanged, one obtains

$$
\begin{aligned}
\hat{\Delta}_{3}(p, q)= & \frac{\pi}{4(2 \pi)^{2}}\left\{-\frac{\operatorname{sgn}\left(P^{0}\right) \Theta\left(P^{2}\right)}{\sqrt{N}} \log \left|\frac{p q+\sqrt{N}}{p q-\sqrt{N}}\right|+\right. \\
& +\frac{\operatorname{sgn}\left(q^{0}\right) \Theta\left(q^{2}\right)}{\sqrt{N}} \log \left|\frac{p^{2}-p q+\sqrt{N}}{p^{2}-p q-\sqrt{N}}\right|+ \\
& \left.-\frac{\operatorname{sgn}\left(p^{0}\right) \Theta\left(p^{2}\right)}{\sqrt{N}} \log \left|\frac{q^{2}-p q+\sqrt{N}}{q^{2}-p q-\sqrt{N}}\right|\right\} .
\end{aligned}
$$

Note that all imaginary parts of the integrals appearing in Equation (40) mutually cancel, since $\hat{\Delta}_{3}$ is real due to its antisymmetry properties in configuration space described above.

\section{Calculation of $\hat{\Delta}_{3}^{\text {ret }}$}

In close analogy to Equation (21), $\hat{\Delta}_{3}^{\text {ret }}(p, q)$ is calculated in the following for $p \in$ $V^{+}$and $q \in V^{-}$. The result for arbitrary $p, q$ can be obtained by analytic continuation, since $\hat{\Delta}_{3}(p, q)$ is the boundary value of an analytic function, regular in 
$p \in \mathbb{R}^{4}+i V^{+}, q \in \mathbb{R}^{4}+i V^{-}$(see also [10]). The procedure is in two steps. First, the integral

$$
\hat{\Delta}_{3, p}(p, q)=\frac{i}{2 \pi} \int_{-\infty}^{+\infty} \mathrm{d} t \frac{\hat{\Delta}_{3}(t p, q)}{1-t+i 0}
$$

is evaluated for $p \in V^{+}$. This corresponds to the calculation of $\Theta\left(x_{1}^{0}-x_{3}^{0}\right)$ $\Delta_{3}\left(x_{1}, x_{2}, x_{3}\right)$ in configuration space in the Lorentz system where $\vec{p}=\left(p^{0}, \overrightarrow{0}\right)$. Then $\hat{\Delta}_{3}^{\text {ret }}(p, q)$ is obtained by calculating

$$
\begin{aligned}
\hat{\Delta}_{3}^{\text {ret }}(p, q) & =\frac{i}{2 \pi} \int_{-\infty}^{+\infty} \mathrm{d} t \frac{\hat{\Delta}_{3, p}(p, t q)}{1-t+i 0} \\
& =-\frac{1}{(2 \pi)^{2}} \int_{-\infty}^{+\infty} \mathrm{d} t \int_{-\infty}^{+\infty} \mathrm{d} s \frac{\hat{\Delta}_{3}(t p, s q)}{(1-t+i 0)(1-s+i 0)},
\end{aligned}
$$

corresponding to the multiplication of $\Theta\left(x_{1}^{0}-x_{3}^{0}\right) \Delta_{3}\left(x_{1}, x_{2}, x_{3}\right)$ with $\Theta\left(x_{2}^{0}-x_{3}^{0}\right)$ in configuration space. Note that the well-known splitting formula [1]

$$
\Delta_{3}^{\mathrm{ret}}(p, q)=\frac{i}{2 \pi} \int_{-\infty}^{+\infty} \mathrm{d} t \frac{\hat{\Delta}_{3}(t p, t q)}{1-t+i 0}
$$

is infrared divergent in the case of a totally massless vertex, while the double dispersion relation Equation (51) is not.

For the actual calculations one may start by considering the first logarithm in Equation (49)

$$
I_{1, p}(p, q)=-\frac{i \pi}{4(2 \pi)^{3}} \int_{-\infty}^{+\infty} \mathrm{d} t \frac{\operatorname{sgn}\left(t p^{0}-q^{0}\right) \Theta\left((t p-q)^{2}\right)}{(1-t+i 0)|t| \sqrt{N}} \log \left|\frac{t p q+|t| \sqrt{N}}{t p q-|t| \sqrt{N}}\right| .
$$

The real part of the integral (53) can be written down immediately $(P=p-q)$

$$
\operatorname{Re}\left[I_{1, p}(p, q)\right]=-\frac{\pi^{2}}{4(2 \pi)^{3}} \frac{\operatorname{sgn}\left(P^{0}\right) \Theta\left(P^{2}\right)}{\sqrt{N}} \log \left|\frac{p q+\sqrt{N}}{p q-\sqrt{N}}\right| .
$$

The Lorentz system shall be chosen such that $p \rightarrow\left(p^{0}, \overrightarrow{0}\right)$. Since $p \in V^{+}$(and $q$ arbitrary), one has to evaluate the Cauchy principal value

$$
\operatorname{Im}\left[I_{1, p}(p, q)\right]=-\frac{i \pi}{4(2 \pi)^{3}} \log \left|\frac{p q+\sqrt{N}}{p q-\sqrt{N}}\right| \int_{-\infty}^{+\infty} \mathrm{d} t \frac{\operatorname{sgn}\left(t p^{0}-q^{0}\right) \Theta\left(\left(\left(t p^{0}, \overrightarrow{0}\right)-q\right)^{2}\right)}{t(1-t)} .
$$


From the $\Theta$ distribution, one obtains integral limits via

$$
t^{2} p_{0}^{2}-2 t p^{0} q^{0}+q^{2}=0 \Rightarrow t_{1,2}=\frac{1}{p^{0}}\left(q^{0} \pm \sqrt{q_{0}^{2}-q^{2}}\right)=\frac{1}{p^{0}}\left(q^{0} \pm|\vec{q}|\right),
$$

and taking into account that $\operatorname{sgn}\left(t_{1,2} p^{0}-q^{0}\right)=\operatorname{sgn}( \pm|\vec{q}|)$, one obtains from

$$
\begin{gathered}
-\int_{-\infty}^{t_{2}} \frac{\mathrm{d} t}{t(1-t)}+\int_{t_{1}}^{\infty} \frac{\mathrm{d} t}{t(1-t)}=\log \left|\frac{\left(1-t_{1}\right)\left(1-t_{2}\right)}{t_{1} t_{2}}\right| \\
=\log \left|\frac{\left(p^{0}, \overrightarrow{0}\right)-q^{2}}{q^{2}}\right|=\log \left|\frac{(p-q)^{2}}{q^{2}}\right|
\end{gathered}
$$

the first 'splitting' result

$$
\begin{aligned}
I_{1, p}(p, q)= & \frac{i \pi}{4(2 \pi)^{3} \sqrt{N}} \log \left|\frac{p q+\sqrt{N}}{p q-\sqrt{N}}\right| \log \left|\frac{q^{2}}{(p-q)^{2}}\right| \\
& -\frac{\pi^{2}}{4(2 \pi)^{3}} \frac{\operatorname{sgn}\left(P^{0}\right) \Theta\left(P^{2}\right)}{\sqrt{N}} \log \left|\frac{p q+\sqrt{N}}{p q-\sqrt{N}}\right| .
\end{aligned}
$$

As further step one may calculate

$$
I_{1, p, q}=\frac{i}{2 \pi} \int_{-\infty}^{+\infty} \mathrm{d} t \frac{I_{1, p}(p, t q)}{1-t+i 0} .
$$

The terms generated by the $i 0$-term can be written down immediately

$$
\frac{i \pi^{2}}{4(2 \pi)^{4} \sqrt{N}} \log \left|\frac{p q+\sqrt{N}}{p q-\sqrt{N}}\right| \log \left|\frac{q^{2}}{(p-q)^{2}}\right|-\frac{\pi^{3}}{4(2 \pi)^{4} \sqrt{N}} \log \left|\frac{p q+\sqrt{N}}{p q-\sqrt{N}}\right| .
$$

The remaining non-trivial integrals are

$$
i_{1}=-\frac{\pi}{4(2 \pi)^{4} \sqrt{N}} \log \left|\frac{p q+\sqrt{N}}{p q-\sqrt{N}}\right| \int_{-\infty}^{+\infty} \frac{\mathrm{d} t}{t(1-t)} \log \left|\frac{t^{2} q^{2}}{(p-t q)^{2}}\right|
$$

and

$$
i_{2}=-\frac{i \pi^{2}}{4(2 \pi)^{4} \sqrt{N}} \log \left|\frac{p q+\sqrt{N}}{p q-\sqrt{N}}\right| \int_{-\infty}^{+\infty} \frac{\mathrm{d} t}{t(1-t)} \operatorname{sgn}\left(t P^{0}\right) \Theta\left(t^{2} P^{2}\right) .
$$

Integral $i_{1}$ can easily be solved by the help of the identity

$$
\lim _{R \rightarrow \infty} \int_{-R}^{+R} \mathrm{~d} t \frac{\log |t-\alpha|}{t-\beta}=\frac{\pi^{2}}{2} \operatorname{sgn}(\beta-\alpha)
$$


and one obtains

$$
i_{1}=\frac{\pi^{3}}{4(2 \pi)^{4} \sqrt{N}} \log \left|\frac{p q+\sqrt{N}}{p q-\sqrt{N}}\right|
$$

i.e. $i_{1}$ cancels the second term in Equation (60). Integral $i_{2}$ has a structure completely analogous to Equation (55) and is given by

$$
\frac{i \pi^{2}}{4(2 \pi)^{3} \sqrt{N}} \log \left|\frac{p q+\sqrt{N}}{p q-\sqrt{N}}\right| \log \left|\frac{p^{2}}{(p-q)^{2}}\right|
$$

such the compact result $\left(p \in V^{+}, q \in V^{-}\right)$

$$
I_{1, p, q}=\frac{i \pi^{2}}{4(2 \pi)^{4} \sqrt{N}} \log \left(\frac{p q+\sqrt{N}}{p q-\sqrt{N}}\right) \log \left(\frac{p^{2} q^{2}}{(p-q)^{2}}\right)
$$

is obtained.

The integrals which appear in the calculation of the remaining parts of $\hat{\Delta}_{3}^{\text {ret }}(p, q)$, namely $I_{2, p, q}$ and $I_{3, p, q}$, can all be expressed by the help of the Spence function (or dilogarithm) $\operatorname{Li}(z)$, which is defined by

$$
\operatorname{Li}(z)=\int_{0}^{z} \frac{\mathrm{d} t}{t} \log (1-t)
$$

where the integral contour in eq. (67) must be equivalent to a straight line from 0 to $z$ avoiding $[1,+\infty) \subset \mathbb{R}$. This is due to the fact that all relevant integrals can be reduced directly to the form $(a, b, c, d \in \mathbb{R})$

$$
\int^{x} \mathrm{~d} t \frac{\log |a t+b|}{c t+d}=-\frac{1}{c} L\left(\frac{a(c x+d)}{a d-b c}\right)+\frac{1}{c} \log \left|\frac{a d-b c}{c}\right| \log \left|\frac{a(c x+d)}{a d-b c}\right|,
$$

where $L(x)$ is the real Spence function, defined for real arguments $x \in \mathbb{R}$ by

$$
L(x)=\frac{1}{2}(L i(x+i 0)+L i(x-i 0)) .
$$

For the sake of brevity, the results are presented here

$$
\begin{aligned}
I_{2, p}(p, q) & =\frac{i \pi}{4(2 \pi)^{3}} \int_{-\infty}^{+\infty} \mathrm{d} t \frac{\operatorname{sgn}\left(q^{0}\right) \Theta\left(q^{2}\right)}{(1-t+i 0)|t| \sqrt{N}} \log \left|\frac{t^{2} p^{2}-t p q+|t| \sqrt{N}}{t^{2} p^{2}-t p q-|t| \sqrt{N}}\right| \\
& =\frac{\pi^{2}}{4(2 \pi)^{3}} \frac{\operatorname{sgn}\left(q^{0}\right) \Theta\left(q^{2}\right)}{\sqrt{N}} \log \left|\frac{p^{2}-p q+\sqrt{N}}{p^{2}-p q-\sqrt{N}}\right|-\frac{i \pi^{3}}{4(2 \pi)^{3}} \frac{\Theta\left(q^{0}\right) \Theta\left(q^{2}\right) \Theta\left(-P^{2}\right)}{\sqrt{N}},
\end{aligned}
$$




$$
\begin{aligned}
I_{2, p, q}(p, q)=-\frac{i \pi^{2}}{4(2 \pi)^{4} \sqrt{N}}[ & 2 L i\left(\frac{p^{2}-p q-\sqrt{N}}{-p q-\sqrt{N}}\right)-2 L i\left(\frac{p^{2}-p q+\sqrt{N}}{-p q+\sqrt{N}}\right)+ \\
& \left.+\log ^{2}\left(\frac{-p q+\sqrt{N}}{p^{2}}\right)-\log ^{2}\left(\frac{-p q-\sqrt{N}}{p^{2}}\right)\right]+ \\
& -\frac{\pi^{3}}{4(2 \pi)^{4} \sqrt{N}}\left[\log \left(\frac{p q-p^{2}-\sqrt{N}}{p q-p^{2}+\sqrt{N}}\right)+\right. \\
& \left.+\log \left(\frac{p q-q^{2}-\sqrt{N}}{p q-q^{2}+\sqrt{N}}\right)+\log \left(\frac{p q+\sqrt{N}}{p q-\sqrt{N}}\right)\right],
\end{aligned}
$$

and $I_{3, p, q}$ ist given by $I_{3, p, q}(p, q)=I_{2, p, q}(q, p)$. Note that the last three logarithmic terms in Equation (71) are superfluous. They indeed appear in the actual calculations; however, they combine to zero, since

$$
\left(\frac{p q-p^{2}-\sqrt{N}}{p q-p^{2}+\sqrt{N}}\right)\left(\frac{p q-q^{2}-\sqrt{N}}{p q-q^{2}+\sqrt{N}}\right)\left(\frac{p q+\sqrt{N}}{p q-\sqrt{N}}\right)=1 .
$$

It must also be pointed out that the Spence function obeys very many identities. For example, the real Spence function satisfies

$$
\begin{aligned}
L(1-x) & =-L(x)+\log |x| \log |1-x|-\frac{\pi^{2}}{6} \\
& =L(1 / x)-\frac{1}{2} \log ^{2}|x|+\log |x| \log |1-x|+\left\{\begin{array}{l}
-\pi^{2} / 3: x<0 \\
+\pi^{2} / 6: x>0
\end{array}\right. \\
& =-L(1-1 / x)+\frac{1}{2} \log ^{2}|x|+\left\{\begin{array}{r}
-\pi^{2} / 2: x<0 \\
0: x>0
\end{array} .\right.
\end{aligned}
$$

Looking for a compact expression for $\hat{\Delta}_{3}^{\text {ret }}$, one finds

$$
\begin{aligned}
\hat{\Delta}_{3}^{\mathrm{ret}}(p, q)=\sum_{i=1}^{3} I_{i, p, q}(p, q) & \\
=\frac{i \pi^{2}}{2(2 \pi)^{4} \sqrt{N}}[ & 2 \operatorname{Li}\left(\frac{p^{2}-p q-\sqrt{N}}{p^{2}}\right)-2 \operatorname{Li}\left(\frac{p^{2}-p q+\sqrt{N}}{p^{2}}\right)+ \\
& \left.+\log \left(\frac{p q+\sqrt{N}}{p q-\sqrt{N}}\right) \log \left(\frac{p^{2}}{(p-q)^{2}}\right)\right] .
\end{aligned}
$$

This expression can be transformed with the help of Equation (73) and the observation that the arguments of the Spence function can be written in many different ways

$$
\begin{aligned}
& \frac{p^{2}-p q-\sqrt{N}}{-p q-\sqrt{N}}=1-\frac{p q-\sqrt{N}}{q^{2}}=1-\frac{p^{2}}{p q+\sqrt{N}} \\
& \frac{p^{2}-p q+\sqrt{N}}{-p q+\sqrt{N}}=1-\frac{p q+\sqrt{N}}{q^{2}}=1-\frac{p^{2}}{p q-\sqrt{N}} .
\end{aligned}
$$


Note that $\hat{\Delta}_{3}^{\text {ret }}(p, q)$ is purely imaginary for $p \in V^{+}, q \in V^{-}$. This insight can also be obtained directly from the naive splitting formula Equation (52), where only the imaginary part is infrared divergent. There, one obtains for the real part of $\hat{\Delta}_{3}^{\text {ret }}(p, q)$ [see also Equation (71)]

$$
-\frac{2 \pi^{3}}{4(2 \pi)^{4} \sqrt{N}}\left[\log \left(\frac{p q-p^{2}-\sqrt{N}}{p q-p^{2}+\sqrt{N}}\right)+\log \left(\frac{p q-q^{2}-\sqrt{N}}{p q-q^{2}+\sqrt{N}}\right)+\log \left(\frac{p q+\sqrt{N}}{p q-\sqrt{N}}\right)\right]=0 .
$$

The retarded distribution $\hat{\Delta}_{3}^{\text {ret }}(p, q)$ can be obtained for arbitrary $p, q$ from Equation (74) by analytic continuation, since $\hat{\Delta}_{3}^{\text {ret }}(p, q)$ is the boundary value of the corresponding function analytic for $p \in \mathbb{R}^{4}+i V^{+}$and $q \in \mathbb{R}^{4}+i V^{-}$. Furthermore, the full vertex function is finally obtained from Equations $(35,36)$

$$
\hat{V}(p, q)=\hat{\Delta}_{3}^{\mathrm{ret}}(p, q)-\hat{\Delta}_{3}^{-}(p, q),
$$

with

$$
\begin{aligned}
\hat{\Delta}_{3}^{-}(p, q)= & \frac{\pi}{4(2 \pi)^{2}}\left\{\left(\frac{\Theta\left(-P^{0}\right) \Theta\left(P^{2}\right)}{\sqrt{N}} \log \left(\frac{p q+\sqrt{N}}{p q-\sqrt{N}}-i 0\right)+\right.\right. \\
& +\frac{\Theta\left(q^{0}\right) \Theta\left(q^{2}\right)}{\sqrt{N}} \log \left(\frac{p^{2}-p q+\sqrt{N}}{p^{2}-p q-\sqrt{N}}-i 0 p^{2}\right)+ \\
& \left.+\frac{\Theta\left(-p^{0}\right) \Theta\left(p^{2}\right)}{\sqrt{N}} \log \left(\frac{q^{2}-p q+\sqrt{N}}{q^{2}-p q-\sqrt{N}}-i 0 q^{2}\right)\right\} .
\end{aligned}
$$

The result for $\hat{\Delta}_{3}^{-}(p, q)$ is given here with the remark that $\hat{\Delta}_{3}^{-}(p, q)$ and $\hat{\Delta}_{3}(p, q)$ can indeed be calculated in parallel. A detailed calculation of $\hat{\Delta}_{3}^{ \pm}$and $\hat{\Delta}_{3}$ can also be found in [1], Chapter 3.8, where the vertex function appearing in QED with massive electrons is treated. The result for the massless case can be easily obtained by setting the electron mass equal to zero, since no infrared problems are present in $\hat{\Delta}_{3}^{ \pm}$and $\hat{\Delta}_{3}$.

For $p \in V^{+}$and $q \in V^{-}, \hat{\Delta}_{3}^{-}(p, q)$ vanishes and one can finally write down in a 'sloppy' style the result that can be found in the literature $[3,6,14]$

$$
\begin{aligned}
\int & \frac{\mathrm{d}^{4} k}{\left[(p-k)^{2}\right]\left[(q-k)^{2}\right]\left[k^{2}\right]} \\
= & \frac{i \pi^{2}}{2 \sqrt{N}}\left[2 L i\left(\frac{p^{2}-p q-\sqrt{N}}{p^{2}}\right)-2 L i\left(\frac{p^{2}-p q+\sqrt{N}}{p^{2}}\right)+\right. \\
& \left.+\log \left(\frac{p q+\sqrt{N}}{p q-\sqrt{N}}\right) \log \left(\frac{p^{2}}{(p-q)^{2}}\right)\right]
\end{aligned}
$$

Note that in the result given in [6], a factor of two is missing. Equation (79) is also equivalent to the forms derived in $[15,16]$. 


\section{Acknowledgements}

This work was supported by the Swiss National Science Foundation.

\section{References}

1. Scharf, G.: Finite Quantum Electrodynamics, 2nd ed In: Springer, Berlin Heidelberg New York (1995)

2. Epstein, H., Glaser, V.: The role of locality in perturbation theory. Ann. Poincaré Phys. Theor. A19, 211 (1973)

3. Davydychev, A.I., Osland, P., Tarasov, O.V.: Three-gluon vertex in arbitrary gauge and dimension. Phys. Rev. D54, 4807 (1996)

4. Streater, R.F., Wightman, A.S.: PCT, Spin, Statistics and All That. Benjamin-Cummings, Company (1964)

5. 't Hooft, G., Veltman, M.J.G.: Scalar one loop integrals. Nucl. Phys. B153, 365 (1979)

6. Ball, J.S., Chiu, T.-W.: Analytic properties of the vertex function in gauge theories. I. Phys. Rev. D22, 2542 (1980)

7. Davydychev, A.I., Delbourgo, R.: A geometrical angle on Feynman integrals. J. Math. Phys. 39, 4299 (1998)

8. Constantinescu, F.: Distributions and Their Applications in Physics. Pergamon, New York (1980)

9. Aste, A.: Dispersive calculation of the massless multi-loop sunrise diagram. Lett. Math. Phys. 77, 209 (2006). DOI 10.1007/s11005-006-0090-6, hep-th/0604113

10. Reeds, M., Simon, B.: Methods of Modern Mathematical Physics, vol. 2, p. 23 and problem 23, p. 124. Academic, New York (1978)

11. Aste, A.: The two loop master diagram in the causal approach. Ann. Phys. 257, 158 (1997)

12. Aste, A., Trautmann, D.: Finite calculation of divergent selfenergy diagrams. Can. J. Phys. 81, 1433 (2003)

13. Aste, A., Scharf, G., Walther, U.: Power counting degree versus singular order in the Schwinger model. Nuovo Cim. A111, 323 (1998)

14. Kizilersü, A., Reenders, M., Pennington, M.R.: One-loop QED vertex in any covariant gauge: Its complete analytic form. Phys. Rev. D52, 1242 (1995)

15. Davydychev, A.I., Usyukina, N.I.: Recursive algorithm of evaluating vertex type Feynman integrals. J. Phys. A25, 5587 (1992)

16. Davydychev, A.I., Usyukina, N.I.: An approach to the evaluation of three and four point ladder diagrams. Phys. Lett. B298, 363 (1993) 